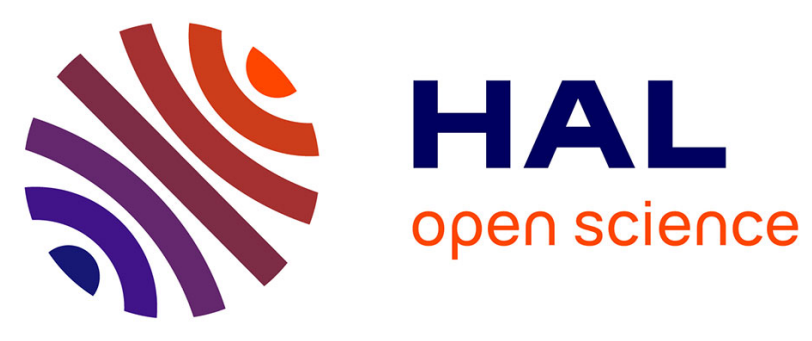

\title{
New laboratory measurements of CH4 in Titan's conditions and a reanalysis of the DISR near-surface spectra at the Huygens landing site
}

David Jacquemart, Emmanuel Lellouch, Bruno Bézard, Catherine de Bergh, Athena Coustenis, Nelly Lacome, Bernard Schmitt, M. Tomasko

\section{To cite this version:}

David Jacquemart, Emmanuel Lellouch, Bruno Bézard, Catherine de Bergh, Athena Coustenis, et al. New laboratory measurements of CH4 in Titan's conditions and a reanalysis of the DISR nearsurface spectra at the Huygens landing site. Planetary and Space Science, 2008, 56 (5), pp.613-623. 10.1016/j.pss.2007.10.008 . hal-00746033

\section{HAL Id: hal-00746033 \\ https://hal.sorbonne-universite.fr/hal-00746033}

Submitted on 26 Oct 2012

HAL is a multi-disciplinary open access archive for the deposit and dissemination of scientific research documents, whether they are published or not. The documents may come from teaching and research institutions in France or abroad, or from public or private research centers.
L'archive ouverte pluridisciplinaire HAL, est destinée au dépôt et à la diffusion de documents scientifiques de niveau recherche, publiés ou non, émanant des établissements d'enseignement et de recherche français ou étrangers, des laboratoires publics ou privés. 


\title{
New laboratory measurements of $\mathrm{CH}_{4}$ in Titan's conditions and a reanalysis of the DISR near-surface spectra at the Huygens landing site
}

\author{
D. Jacquemart ${ }^{1}$, E. Lellouch², B. Bézard², C. de Bergh ${ }^{2}$, \\ A. Coustenis ${ }^{2}$, N. Lacome ${ }^{1}$, B. Schmitt ${ }^{3}$, and M. Tomasko ${ }^{4}$
}

${ }^{1}$ Université Pierre et Marie Curie-Paris 6, LADIR, CNRS, UMR 7075, Case courrier 49, Bât F 74, 4, place Jussieu, 75252 Paris Cedex 05, France.

${ }^{2}$ LESIA, Observatoire de Paris, CNRS, Université Pierre et Marie Curie-Paris 6, Université Paris-Diderot, 5 place Jules Janssen, 92195 Meudon, France

${ }^{3}$ LPG, Université Joseph Fourier, 38041, Grenoble, France

${ }^{4}$ LPL, University of Arizona, Tucson, AZ 85721, USA

\begin{abstract}
Laboratory spectra of methane-nitrogen mixtures have been recorded in the near-infrared range $(1.0-1.65 \mu \mathrm{m})$ in conditions similar to Titan's near surface, to facilitate the interpretation of the DISR/DLIS spectra taken during the last phase of the descent of the Huygens Probe, when the surface was illuminated by a surface science lamp. We used a 0.03 $\mathrm{cm}^{-1}$ spectral resolution, adequate to resolve the lines at high pressure ( $\mathrm{p}_{\mathrm{N} 2} \sim 1.5$ bar). By comparing the laboratory spectra with synthetic calculations in the well-studied $v_{2}+2 v_{3}$ band (7515-7620 $\mathrm{cm}^{-1}$ ), we determine a methane absorption column density of $178 \pm 20 \mathrm{~cm}$-am and a temperature of $118 \pm 10 \mathrm{~K}$ in our experiment. From this, we derive the methane absorption coefficients over 1.0-1.65 $\mu \mathrm{m}$ with a $0.03 \mathrm{~cm}^{-1}$ sampling, allowing for the extrapolation of the results to any other methane column density under the relevant pressure and temperature conditions. We then revisit the calibration and analysis of the Titan "lamp-on" DLIS spectra. We infer a $5.1 \pm 0.8 \%$ methane mixing ratio in the first $25 \mathrm{~m}$ of Titan's atmosphere. The $\mathrm{CH}_{4}$ mixing ratio measured $90 \mathrm{sec}$ after landing from a distance of $45 \mathrm{~cm}$ is found to be $0.92 \pm 0.25$ times this value, thus showing no post-landing outgassing of methane in excess of $\sim 20 \%$. Finally, we determine the surface reflectivity as seen from $25 \mathrm{~m}$ and $45 \mathrm{~cm}$ and find that the $1500 \mathrm{~nm}$ absorption band is deeper in the post-landing spectrum as compared to pre-landing.
\end{abstract}




\section{Introduction}

The Descent Imager /Spectral Radiometer (DISR) investigation onboard the Huygens Probe (Tomasko et al. 2002) has revolutionized our understanding of Titan's surface/atmosphere system, revealing traces of past flows on Titan's surface, probably driven by intense and episodic methane rain (Tomasko et al. 2005). Beyond this spectacular discovery, DISR also contributed in characterizing essential aspects of Titan's atmosphere, such as the distribution and properties of the haze (particle size, optical depth) and the wind profile as a function of altitude below $150 \mathrm{~km}$. In addition to direct imaging, surface science with DISR included measurements of the surface spectrum. For this purpose, the DISR downward-looking spectrometers (DLVS and DLIS), operating respectively at 500-980 nm and 840-1700 nm, were associated with a $20 \mathrm{~W}$ surface science lamp (SSL) which was turned on in the last phase of the descent. The SSL illuminated the surface, providing light even in strong methane bands where solar radiation had been completely absorbed in Titan's atmosphere. Although the lamp was turned on near $620 \mathrm{~m}$ altitude, about 136 seconds before landing, its signal became visible only below about $60 \mathrm{~m}$ from the surface. Between this altitude and the landing, two DLVS and two DLIS spectra were recorded. After landing, DLIS and DLVS continued to acquire surface spectra from a distance of about $50 \mathrm{~cm}$.

Even from an altitude of only a few tens of meters, and at the low resolution of the DISR spectrometers, methane absorption is clearly visible in the lamp spectra through signatures centred at $890,1000,1120-1160$, and 1340-1420 nm. Retrieving a methane abundance from these features requires an appropriate knowledge of the methane absorption properties. Methane spectroscopy in the near-infrared (shortward of $1600 \mathrm{~nm}$ ) is insufficiently known, as line-by-line information necessary for synthetic spectra calculations in any temperature, path, and pressure conditions (i.e. including line position, intensity, and lower energy level) is restricted to a few narrow intervals. This problem, which has in fact hindered analyses of near-infrared spectra of the giant planets and Titan for several decades, is usually being circumvented by the use of low-resolution "absorption coefficients". These are obtained from band models fit to laboratory data and recast in the form of exponential-sum coefficients. In effect, this approach accommodates the fact that at low resolution, the absorption does not follow Beer's law. Still, as demonstrated in Fig. 3 of Sromovsky et al. (2006), no laboratory measurements, even at low spectral resolution, yet sample the full range of pressurestemperatures- $\mathrm{CH}_{4}$ paths relevant to outer planet conditions. For the analysis of the entire DISR dataset, Tomasko et al. (2005) used absorption coefficients based on the results of Karkoschka (1998) at wavelengths shorter than $1050 \mathrm{~nm}$ and on a modified version of the Strong et al. (1993) Goody-Voigt model at longer wavelengths. These exponential-sum coefficients were matched (i.e. spectrally convolved) to the spectral pixels of the DISR spectrometers, and calculated on a temperature/pressure grid appropriate to Titan. With this set of absorption coefficients (referred to as "the Karkoschka model" hereafter), Tomasko et al. (2005) analyzed the highest S/N lamp-on DLIS spectrum -- the last one taken before landing -- and inferred a 5+/-1 \% methane abundance in the first $21 \mathrm{~m}$ of Titan's atmosphere. They also found that the gas transmission model did not provide a perfect match of the absorption bands in the observed lamp spectrum, leaving in particular, residuals in the 1000 $\mathrm{nm}$ band and in the absorption shoulder near $1320 \mathrm{~nm}$.

Recently, new absorption coefficients suitable to low temperature and long path conditions have become available longwards of $1050 \mathrm{~nm}$ (Irwin et al. 2006). They were calculated from a combined reanalysis of the Strong et al. (1993) data and of recent, lower temperature (100140 K) short-path measurements obtained by Sihra (1998). Sromovsky et al. (2006) 
demonstrated the better agreement of the new coefficients with both the laboratory measurements of McKellar (1989) at $77 \mathrm{~K}$ and the near-IR spectrum of Uranus. As discussed later, these coefficients (see http://www.atm.ox.ac.uk/user/irwin/methane.html), when used to calculate the atmospheric transmission in conditions typical of Titan's near surface $(\mathrm{T}=94 \mathrm{~K}$, $\mathrm{p}=1.5$ bar) predict less absorption in the core of the strong 1150 and $1360 \mathrm{~nm}$ than the Karkoschka model, as well as small differences throughout the spectrum. This of course has implications for the retrieved methane abundance.

The overall validity and merits of the existing sets of absorption coefficients has been explored by Negrão et al. (2006), based on a comparison with ground-based observations. Furthermore, they can be tested against the entire DISR spectral dataset, which offers the unique opportunity to probe the atmosphere in successive "slices". Such an analysis, performed by Tomasko et al. (2007), has the ultimate goal of providing the community with reliable methane absorption coefficients in Titan's conditions (albeit at low spectral resolution), a task of utmost importance especially in view of the huge Cassini/VIMS dataset. With the more restricted objective of analyzing the DISR lamp-on surface spectra, another, simpler, approach is to acquire laboratory measurements of the methane absorption properties in conditions as similar as possible to the near surface of Titan, enabling a "direct" comparison with the DISR observations. This is the goal of the present paper. Laboratory measurements of $\mathrm{CH}_{4}-\mathrm{N}_{2}$ mixtures are presented in the next section, and the absorption coefficients are extracted. Results are then applied to the DISR/DLIS spectra acquired just before and after landing, and results on the near-surface methane mixing ratio and surface spectrum are presented.

\section{Laboratory spectra of methane-nitrogen mixtures}

The rapid scan Bruker IFS 120 HR interferometer of the LADIR (Laboratoire de Dynamique, Interactions et Réactivité) was used to record laboratory spectra of methane-nitrogen mixtures, using a maximal optical path difference of $18 \mathrm{~cm}$. The interferometer was equipped with a $\mathrm{CaF}_{2} / \mathrm{Si}$ beamsplitter, an InGaAs detector, and a Tungsten source. No optical filter was used, so that the covered spectral region was between 1000 and $1650 \mathrm{~nm}$. For all spectra the whole optical path was under vacuum. A multipass cell of one meter base length was used for a total absorption path of $2015 \pm 1 \mathrm{~cm}$. The cell was equipped with $\mathrm{KCl}$ windows. The commercial methane gas sample, furnished by Air Liquid, with a stated purity of $99.99 \%$ in natural abundances, was used. The temperature of the gas was recorded with four platinum probes located at different places inside the cell. In order to cool the gas, liquid nitrogen was directly injected in the second envelope of the cell. The pressure of the gas was measured with capacitance MKS Baratron manometers. The spectra were not numerically apodized, but they were slightly over sampled (over sampling ratio equal to 2) by post-zero filling the interferograms. Averaging 100 scans, the signal-to-noise ratio (peak to peak) is nearly equal to 200 around $1650 \mathrm{~nm}$, but is decreasing to 20 around $1000 \mathrm{~nm}$. This is mainly due to the response of the InGaAs detector. Note that in the pressure conditions of our spectra (1.5 atm of $\mathrm{N}_{2}$ ), the apparatus-function width $\left(\mathrm{FWHM}=0.033 \mathrm{~cm}^{-1}\right.$ ) is negligible compared to the Lorentz line widths of methane, especially at low temperature (FWHM $\sim 0.4 \mathrm{~cm}^{-1}$ around 100 $\mathrm{K}$ and $0.2 \mathrm{~cm}^{-1}$ around $300 \mathrm{~K}$ ). The $100 \%$ transmission level of the laboratory spectra was derived using spectra recorded with no gas, and in the same temperature and optical conditions. 
We tried to reproduce as closely as possible the Titan surface conditions ( $\mathrm{p}_{\mathrm{N} 2} \sim 1.4$ bar, $\mathrm{p}_{\mathrm{CH} 4} \sim$ 70 mbar, $\mathrm{T} \sim 94 \mathrm{~K}$ ). To record a low temperature spectrum, the cell was first filled at room temperature with methane and $\mathrm{N}_{2}$. Liquid nitrogen was then introduced in the second envelope of the cell. As the gas cools, the pressure in the cell decreases, so that $\mathrm{N}_{2}$ was reinjected by steps until the appropriate experimental conditions were reached. However, several difficulties were encountered in this procedure. Due to direct injection of liquid nitrogen in the secondary envelope of the cell, complete thermalization of the cell was not achieved, leading to a temperature gradient. Indeed, the temperature sensors indicated a gradient of $20 \mathrm{~K}$ ranging from $83 \mathrm{~K}$ (for the sensor closest to the walls of the cell) to $103 \mathrm{~K}$ (for the sensor farthest from the walls). Because there was no temperature sensor in the middle of the cell, the average temperature of $93 \mathrm{~K}$ is certainly not representative of the actual mean temperature of the absorbing methane. Moreover, liquid nitrogen in contact with the external envelope of the cell led to non-negligible condensation of methane. Therefore the effective temperature and partial pressure of methane in the cell had to be determined a posteriori, as discussed below.

In view of a longer-term study of the line parameters of methane, we also acquired a number of spectra at room and intermediate temperatures. As explained below, the room temperature spectrum (500 scans) is of use for the present study. Its conditions are the following: $\mathrm{T}=297$ $\mathrm{K}, P_{\mathrm{CH} 4}=0.1053 \mathrm{~atm}, P_{\mathrm{N} 2}=0.894 \mathrm{~atm}$. Unlike the low temperature spectrum, these conditions are well determined.

\section{Analysis and determination of methane absorption coefficients}

The first step of the analysis consists in determining the actual effective conditions (column density of methane, temperature) in the low temperature spectrum. For this, we compared the measurements with synthetic spectra in selected spectral ranges, based on the HITRAN database (Rothman et al. 2005). As noted above, this and all other databases are extremely incomplete. For example the energy of the lower state (noted E") of the 28226 transitions present between 900 and $1600 \mathrm{~nm}$ is only known for 205 lines. This results from the high number of levels involved, leading to serious difficulties in transition assignments, even for cold bands. This limitation makes it impossible to calculate synthetic spectra at low temperature, except in restricted spectral intervals where assigned transitions are well isolated from other, non-assigned, transitions. We found that the $7515-7620 \mathrm{~cm}^{-1}$ portion of the $v_{2}+2 v_{3}$ band matches this requirement. In this spectral range, 44 assigned transitions are present in the current version of HITRAN and come from the work of Hippler and Quack (Hippler and Quack 2002); their quantum number $J$ " varies from 0 to 9 . Since the temperature dependence of line intensities varies significantly with $J$ ”, this allows one to simultaneously determine the temperature and the column density of methane in the conditions of the spectrum.

Synthetic spectra were calculated using a Lorentz profile. We first validated the calculation by fitting the laboratory spectrum recorded at room temperature. For this, knowing the lower energy levels is not necessary, so the HITRAN database can be used without modification. In the studied spectral range, all transitions in HITRAN have default constant values for broadening coefficients and their temperature dependence. Pressure shifts from $\mathrm{N}_{2}$ cannot be neglected at the resolution of our measurements, and the pressure-shift coefficient of HITRAN (Rothman et al. 2004), about $-0.014 \mathrm{~cm}^{-1} \mathrm{~atm}^{-1}$ between 7520 and $7620 \mathrm{~cm}^{-1}$, was used. As can be seen in Fig. 1 (top panel), the synthetic spectrum (red curve) matches almost 
perfectly the experimental spectrum (black curve) recorded at $297 \mathrm{~K}$ with $0.1053 \mathrm{~atm}$ of methane and 0.894 atm of $\mathrm{N}_{2}$. For comparison, the calculation with only the assigned transitions from HITRAN is plotted as the green line in Fig. 1 (top).

One can observe that some of the transitions are not assigned. It is the case in particular of some lines around 7586, 7595, 7609, and $7618 \mathrm{~cm}^{-1}$, that are observed in spectra at lower temperatures. We tried to estimate their lower energy levels $E^{\prime \prime}$ from low temperature spectra. In the studied spectral region, the $v_{2}+2 v_{3}$ band is well isolated, so we can assume that transitions with the same value of $J^{\prime \prime}$ (same value of $E^{\prime \prime}$ ) lie close to each other. This assumption seems to be confirmed by a new spectroscopic analysis of this region (Rey, Boudon, and Quack, private communication). We estimated the lower states of 45 transitions as having $J^{\prime \prime}$ values between 1 to 9; some of them are certainly from the $v_{2}+2 v_{3}$ band, while others may belong to other weaker cold bands, such as the $3 v_{2}+v_{3}$ band (same priv. comm.). No true assignment has been performed in this work; we focused only on estimating the lower energy level of the transitions. Note that most of these new "assigned" transitions are quite weak, but will improve the global modelling of the low temperature spectrum. The line positions, intensities, and lower energy levels of the assigned transitions present in HITRAN, plus the line positions and intensities from HITRAN of the 45 transitions for which the lower energy level has been estimated, constitute what we will call HITRAN*. For this reduced line list, the default values for $\mathrm{N}_{2}$-broadening coefficients and their temperature dependence were replaced by values depending on $J$ ". The $\mathrm{N}_{2}$-broadening coefficients were taken from Perrin and Soufiani (2007), except for $J "=0,1$ and 2, for which average data of Pine and Gabard (2003) were used. Concerning the temperature exponent, values between 0.9 and 0.7 were used for values of $J$ " between 0 to 9. Calculation at room temperature using HITRAN* transitions is plotted in green in Fig. 1(b); for comparison, a calculation including in addition the non-assigned transitions from HITRAN is shown in red.

We then turned to the low temperature spectrum. A grid of calculations using HITRAN* and various column densities and temperatures of methane is plotted on Fig. 2, demonstrating how the column density of methane and the temperature can be retrieved simultaneously. Using HITRAN* instead of HITRAN allowed to better model the groups of lines with $J$ ” $=0$ to 9 . As can be seen from the residuals in Fig. 2, the line list is still not perfect. The best fit solution is obtained for a column density of $178 \mathrm{~cm}$ am, and a temperature of $118 \mathrm{~K}$ (corresponding to a methane partial pressure of $0.038 \mathrm{~atm}$ ). Note that the pressure of $0.038 \mathrm{~atm}$ corresponds to the vapour pressure at a temperature equal to $84 \mathrm{~K}$ (Prialnik et al. 2005), close to the coldest temperature in the cell. However, due to the absolute uncertainty of our temperatures measurements $( \pm 3 \mathrm{~K})$, no accurate estimation of methane pressure can be performed using the lowest recorded temperature. We estimate an uncertainty of $\pm 11 \%$ on the column density and $\pm 10 \mathrm{~K}$ on the temperature. These uncertainties may seem a little conservative given the grid of models of Fig. 2, but we here also account for the effect of the uncertainty on the 100 $\%$ transmission level. Note that between 7515 and $7520 \mathrm{~cm}^{-1}$, no lines are assigned in HITRAN or HITRAN*, which explains why the calculation in Fig. 2 does not reproduce the experimental spectrum in this spectral range.

The low-temperature spectrum shows 4 absorption regions due to methane. However, only the 1100-1200 and 1300-1500 nm regions (respectively the triacontad and the icosad) will be used. The tetradecad region at 1650-1900 nm (on the edge of the detection possibilities for the InGaAs detector) falls outside of the DISR range. Fig. 3 shows the $900-1600 \mathrm{~nm}$ portion of the spectrum. The $1000 \mathrm{~nm}$ absorption region is also not used because of insufficient signal-to-noise in the laboratory spectrum. At wavelengths where the methane absorption is 
not unambiguously detected, the transmission is fixed to 1 (see Fig. 3). As mentioned previously, the instrumental convolution is negligible, so the measured transmission can be at each frequency converted to an absorption coefficient using Beer’s law.

\section{Application to DISR spectra}

\section{a. Data selection and calibration}

As discussed in Tomasko et al. (2005), nine DLIS spectra with the Surface Science Lamp (SSL) on were recorded below $700 \mathrm{~m}$ altitude. Each of these spectra has a sampling time of 1.00 sec. Following Schröder and Keller (2007), we refer to these spectra by using their DISR internal number (\#), mission time, and/or altitude, according to the trajectory reconstruction of Karkoschka et al. (2007) (see Table 1). As outlined in Tomasko et al. (2005), as soon as the lamp was turned on, the spectra showed an increase of signal at all wavelengths, due to scattered light from the lamp within the instrument. In addition, all spectra show a large contribution due to the diffuse solar field. This component actually dominates over the signal due to the lamp reflection from the surface at all wavelengths except in the strong methane bands. Only two (\# 206 and \# 210) out of the nine spectra show significant lamp signal reflected off the surface. They were acquired respectively at 55 and $24.8 \mathrm{~m}$ above the surface, where the altitude corresponds to the middle of the exposure. We focus on spectrum \# 210, in which the surface signal is $\sim 5$ times stronger. To extract a "lamp-only" spectrum, we essentially followed the procedure outlined in Tomasko et al. (2005), on which we elaborate here, with some minor differences.

In order to eliminate the instrument-scattered light and the diffuse solar light, the simplest approach is to subtract a "background" spectrum from the spectrum of interest. Ideally, the background spectrum should be a "lamp-off" spectrum of the same area taken at the same altitude and with the same solar azimuth with respect to the center of the instrument field-ofview. As this is not possible in practice, we proceeded empirically by exploring combinations of the other lamp-on spectra that would provide an "optimum" background. Because the solar light dominates so much over the lamp signal in regions of weak methane absorptions, in particular near 1130 and $1280 \mathrm{~nm}$, a criterion for selecting the background was the requirement that the background-subtracted spectrum should be reasonably flat in these spectral regions, as it should be if all solar light has been properly eliminated. We found that spectra \# 199 and \# 202, taken respectively at 109 and $86 \mathrm{~m}$ altitude, provided appropriate background, and consistent with Tomasko et al. (2005) that the mean of these two spectra allowed an optimum correction of the solar field. Fig. 4 illustrates the sensitivity of the resulting lamp-only spectrum to slightly different choices. Note that Schröder and Keller (2007) used instead spectrum \# 202 multiplied by 1.01 for background reference.

Once recovered in this manner, the lamp-only spectrum must be divided by the spectral response of the SSL, which has been measured on the ground during instrumental calibration. We use here the relative spectral response derived by Schröder and Keller (2007) from a spline fit of the original measurements and correction for an absorption band at $1380 \mathrm{~nm}$, caused by atmospheric water. This SSL spectrum also does not show a $1280 \mathrm{~nm}$ absorption that was present in the SSL response used by Tomasko et al. (2005), and shows a little more decrease of power from 800 to $1600 \mathrm{~nm}$. 
For calibration of the lamp-only Titan spectrum in terms of an I/F reflectivity, we did not attempt to use the absolute SSL fluxes measured in the laboratory, as Schröder and Keller (2007) did. Instead, we followed and improved on the method used by Tomasko et al. (2005). It consists of calculating the $\mathrm{I} / \mathrm{F}$ reflectivity in regions of negligible methane absorption as the ratio of the upward intensity to the downward flux $(\pi \mathrm{F})$ divided by $\pi$. The upward intensity is determined from the DLIS measurements, averaged over all lamp-on spectra except \# 210 (i.e. spectra \# 194, 196, 199, 202, 204 and 206). To estimate F, Tomasko et al. (2005) averaged the two ULIS-measured spectra (among the nine taken in the last phase of the descent) having the strongest and the weakest intensity, respectively. As azimuth information is now available (Table 1), this approach can be refined. We averaged the three ULIS spectra with the Sun in the field-of-view (\# 202, 206, and 210, taken at 86, 55 and $24.8 \mathrm{~m}$, and with azimuths of 322, 5 and $45^{\circ}$ respectively). This yielded a mean spectrum $S_{1}$. We also averaged the two ULIS spectra with the Sun most distant from the FOV (\# 190 and 194, taken at 708 and $200 \mathrm{~m}$, and with azimuths of 146 and $166^{\circ}$ ), yielding a mean spectrum $\mathrm{S}_{2}$. Based on a set of synthetic calculations, including the spatial response of the ULIS diffuser, we found that the combination $0.4 \times \mathrm{S}_{1}+0.6 \times \mathrm{S}_{2}$ provided a very good approximation to $\mathrm{F}$. Compared to the initial analysis of Tomasko et al. (2005), the slight change in the relative weights given to the "Sun" and "no-Sun" spectra can be understood from the ULIS diffuser response vs. zenith angle. With the DLIS and ULIS data used in this way, we finally determined I/F reflectivities of $0.166,0.165,0.155,0.140$ and 0.727 at wavelengths of $837,938,1079,1282$, and 1553 $\mathrm{nm}$, with an estimated precision of $\pm 10 \%$. As illustrated in Fig. 4, scaling the lamp-only spectrum by a constant factor provided a satisfactory match of these continuum reflectivities, and we will onwards refer to this calibrated spectrum as "the spectrum at $25 \mathrm{~m}$ ". Beyond the methane absorptions, the spectrum shows a significant blue slope, although somewhat less than in its version originally shown by Tomasko et al. (2005), a result of the newly adopted relative spectral response of the SSL. As compared to the spectrum presented by Schröder and Keller (2007), the main difference is in the absolute calibration, as our I/F reflectivities are about $40 \%$ lower than theirs. The origin of this discrepancy is uncertain, although Schröder and Keller speculate on a possible phase angle dependence of the surface reflectivity.

We also considered DLIS spectra taken after landing. Specifically, we reanalyzed the first non-overexposed spectrum (\# 249), recorded about $90 \mathrm{sec}$ after landing and with a sampling time of 16 msec. About 20 other spectra were taken subsequently, but they all appear to be identical. Spectrum \# 249 was corrected with the same "background" spectrum as spectrum \# 210 , and divided by the SSL relative spectral response. Because the lamp signal from a distance of $\sim 50 \mathrm{~cm}$ is so overwhelmingly dominant over the solar light, the background correction is in fact insignificant. For absolute calibration, we simply rescaled the spectrum to the $\mathrm{I} / \mathrm{F}$ continuum values of the spectrum at $25 \mathrm{~m}$, referring to the result as "the post-landing spectrum”. The 1050-1250 nm range of this spectrum is shown in Fig. 5, and Fig. 6 shows the entire post-landing spectrum after correction of its methane absorption.

b) Determination of $\mathrm{CH}_{4}$ near-surface mixing ratio and surface reflectance spectrum

\section{Method}

Determining the $\mathrm{CH}_{4}$ mixing ratio in the Titan spectra from the laboratory spectra is straightforward in principle. The laboratory spectra allow one to determine "monochromatically" (i.e. at the resolution of the laboratory spectra) the absorption coefficient (in $\mathrm{cm}^{-1}-\mathrm{am}^{-1}$ ) as a function of wavelength, $k_{\lambda}$, from $k_{\lambda}=-1 / a \ln \left(\mathrm{T}_{\lambda}\right)$, where $a$ is the methane column density in the laboratory spectrum $(178 \mathrm{~cm}$-am). The "monochromatic" 
transmittance for any column $b$ is then calculated as $\mathrm{T}_{\lambda}^{\prime}=\exp \left(-k_{\lambda} b\right)$, and convolved by the DLIS instrumental function, which can be approximated with a Gaussian function with $15 \mathrm{~nm}$ FWHM. The methane column $b$ is adjusted until agreement with the $\mathrm{CH}_{4}$ bands in the Titan spectra is reached, and converted into a methane near-surface mixing ratio $q$ (i.e. the ratio of the number of methane molecules per unit volume to the total number of molecules per unit volume at the surface level, also known as the mole fraction). Due to the short-wavelength cutoff of the laboratory spectra, we restrict ourselves to the 1050-1600 nm range. As the absorption in the lamp spectra is two-way and the DLIS spectrometer looks at a nadir angle of $21.4^{\circ}$, the absorption length is $L=2 \mathrm{z} / \cos \left(21.4^{\circ}\right)$, where $z$ is the altitude of the spectrum of interest. $q$ is related to $b$ through to $b=q \times p \times 273.15 / T \times L$, where $\mathrm{p}=1.47$ bar and $\mathrm{T}=93.7$ $\mathrm{K}$ at Titan's surface (Fulchignoni et al. 2005).

\section{Post-landing spectrum}

Tomasko et al. (2005) noted that the post-landing spectrum shows hints for methane absorption features but did not attempt to model them in the absence of information on the absorption path length. Based on the Karkoschka et al. (2007) analysis, the DLIS is at $45 \mathrm{~cm}$ above the surface after the landing. With this distance, the clearly detected $1150 \mathrm{~nm} \mathrm{CH}_{4}$ absorption band indicates a methane mixing ratio $q=4.7 \pm 0.8 \%(b=19.5 \pm 3.2 \mathrm{~cm}$-am, i.e. 9.1 times less than in the laboratory spectrum) where the uncertainty reflects only fitting errors (Fig. 5). To fit the band, which superimposes on a blue slope, a simple linear continuum was assumed. In contrast, the expected 1300-1450 nm absorption is not obviously present in the spectrum, as noted by Schroeder and Keller (2007), and is presumably masked by weak surface reflectance features.

Dividing at all wavelengths the post-landing spectrum by the methane transmission provides the surface reflectivity at the landing point (Fig. 6) from 840 to $1600 \mathrm{~nm}$. Shortward of 1050 $\mathrm{nm}$ (where we do not have reliable laboratory measurements), it can be safely assumed that the methane transmission for such a small path is equal to $100 \%$. The surface reflectivity is similar to the one originally presented by Tomasko et al. (2005), with minor differences due to the different choice of the SSL spectrum (in particular, the new surface spectrum shows a somewhat less blue slope and no emission "bump” near $1280 \mathrm{~nm}$ ) and to this atmospheric correction. Except for a deep absorption longward of $1450 \mathrm{~nm}$, the spectrum is remarkably featureless. The only other (possibly) significant feature is a reflectance maximum near 1360 $\mathrm{nm}$. This feature is present in the post-landing spectrum uncorrected for methane, and is somewhat amplified by the atmospheric correction, as methane has absorption peaks at 1360 and $1400 \mathrm{~nm}$ and a local absorption minimum near $1375 \mathrm{~nm}$.

\section{Spectrum at $25 \mathrm{~m}$}

Figure 7 shows the fit of the spectrum at $25 \mathrm{~m}$ with various $\mathrm{CH}_{4}$ abundances. Such a fit requires an assumption on the surface reflectance, and we first used the reflectance inferred from the post-landing spectrum. However, this approach led to an underestimate of the observed spectrum in its long-wavelength edge. To achieve a good fit, it was necessary to increase the surface reflectance at wavelengths longer than $1420 \mathrm{~nm}$ (Fig. 6), resulting in a slightly shallower $1500 \mathrm{~nm}$ absorption. As the DLIS was looking at different terrains from distances of $25 \mathrm{~m}$ and $45 \mathrm{~cm}$, the difference - which was visible in Fig. 15a of Tomasko et al. (2005) but not discussed - may conceivably be real. The detailed interpretation of the surface spectra, and particularly the origin of the $1500 \mathrm{~nm}$ band, will be presented in Schmitt et al. (2007, in preparation). As noted by Tomasko et al. (2005), this band is reminiscent of water ice absorption, but the identification is not unique. In particular, bright yellow-orange tholins produced in laboratory experiments (and which are therefore plausible candidates on Titan's 
surface) also show absorption in the same range. Whatever the material responsible for this band, a different band depth may indicate a variation of abundance, grain size, and/or state of ice. Beyond this absorption, no significant difference can be found between the surface reflectance as inferred from the spectrum at $25 \mathrm{~m}$ and the post-landing spectrum. In particular, a $1320 \mathrm{~nm}$ mismatch, suggested by Tomasko et al. (2005) on the basis of the "Karkoschka" coefficients does not seem to be significant any more.

With the adopted surface reflectance, the spectrum at $25 \mathrm{~m}$ indicates a best fit $\mathrm{CH}_{4}$ column abundance $b=1165 \pm 90 \mathrm{~cm}$-am, i.e. a mixing ratio of $5.1 \pm 0.4 \%$ (Fig. 7). This column corresponds to an extrapolation by a factor of 6.5 from the laboratory conditions, but this is not a problem because the lines are fully resolved. The error bar is estimated from the fact that optimum fit of the $1150 \mathrm{~nm}$ band is achieved for $4.7 \%$, while the $1400 \mathrm{~nm}$ band would favour a $5.5 \%$ mixing ratio. Additional errors, due to the uncertainty of the methane column density and temperature in the laboratory data, are discussed hereafter. Being systematic, these errors affect the methane determination in the post-landing and the $25 \mathrm{~m}$ spectrum in the same way. Thus the ratio of the post-landing to the pre-landing methane mixing ratio is $0.92+0.25 /-0.22$, i.e., its upper limit is 1.17 . The result is thus not consistent with the GCMS observation of a $40 \%$ increase in the methane mixing ratio over the 2 minutes following the Huygens landing that was presumed to be due to the surface heating by the instrument and attendant evaporation from a liquid methane containing surface. Schroeder and Keller (2007) also find that the pre- and post-landing spectra give consistent amounts of methane.

\section{Uncertainties}

In addition to the $\sim 8 \%$ uncertainty associated with data fitting, the methane surface mixing ratio is affected by the adopted $11 \%$ uncertainty on the methane amount in laboratory data. A third source of uncertainty, more difficult to estimate, comes from the fact that we are using absorption coefficients (in cm-am ${ }^{-1}$ ) determined at $118 \pm 10 \mathrm{~K}$ to model a spectrum at $94 \mathrm{~K}$. To get a handle of the possible resulting error, we performed synthetic calculations on other methane bands for which line-by-line information is complete and reliable. Fig. 8 shows models in the region of the pentad $\left(2400-3300 \mathrm{~cm}^{-1}\right)$ and of the octad $\left(3700-4800 \mathrm{~cm}^{-1}\right)$, at $118 \mathrm{~K}$ and $94 \mathrm{~K}$. The models were convolved to a spectral resolution comparable to that of the DISR/DLIS resolution, and the amount of methane was adjusted in order to get bands of comparable depths than those observed on Titan. Namely, we used $15 \mathrm{~cm}$-am for the pentad, and $80 \mathrm{~cm}$-am for the octad, ensuring a minimum transmission of about 0.1 , as in the Titan spectrum at $25 \mathrm{~m}$. Fig. 8 shows the effect of the gas temperature on the band shape. Overall, the bands are slightly more saturated at $94 \mathrm{~K}$ than at $118 \mathrm{~K}$ (since fewer levels are being populated), which leads to a slightly smaller absorption equivalent width for the same column. Recovering the equivalent width calculated at $118 \mathrm{~K}$ with the synthetic spectrum at $94 \mathrm{~K}$ would require an increase of the $\mathrm{CH}_{4}$ column by $10 \%$ for the pentad and $12.5 \%$ of the octad. This gives an estimate of the maximum error that would result from using absorption coefficients at $118 \mathrm{~K}$ to model an observed spectrum at $94 \mathrm{~K}$ in the region of the pentad and the octad. Note however the strong difference in shape between the $94 \mathrm{~K}$ and $118 \mathrm{~K}$ cases. In the case of Titan, the 1150 and $1400 \mathrm{~nm}$ band shapes are very well reproduced by our $118 \mathrm{~K}$ model. This argues for a much smaller effect than in the region of the icosad and triacontad. Perhaps still the effect is different in the 1150 and $1400 \mathrm{~nm}$ bands, possibly explaining why the two bands are best fit with slightly different $\mathrm{CH}_{4}$ mixing ratios. In an attempt to account for this effect, we include an additional $5 \%$ uncertainty, which is smaller than the other two sources of errors. Altogether, combining the error bars quadratically, we estimate a total $15 \%$ uncertainty on the surface mixing ratio, which is finally estimated to be equal to $5.1 \pm 0.8 \%$. 
For the post-landing spectrum, the individual error bars (17\%, $11 \%$ and $5 \%$ ) combine to produce a $21 \%$ uncertainty, and the final estimate of the methane mixing ratio is $4.7 \pm 1 \%$.

\section{c) Comparison of absorption coefficients}

To try and assess the quality of the absorption coefficients proposed by various authors, we compare in Fig. 9 fits of the $25 \mathrm{~m}$ spectrum using the "Karkoschka" model, the Irwin et al. (2006) coefficients, and the coefficients derived in the present work. For all these models, we use a methane column density of $1165 \mathrm{~cm}$-am. Clearly apparent is the fact that the Karkoschka model, which was used by Tomasko et al. (2005), produces slightly more absorption than the other models, and requires accordingly a 20 \% smaller methane amount to fit the spectrum. (Indeed, Tomasko et al. (2005) determined a $960 \mathrm{~cm}$-am best fit column. This still corresponded to a $5 \pm 1 \%$ mixing ratio, consistent with our present value, because they assumed an altitude of $21 \mathrm{~m}$ for this spectrum). Fig. 9 also shows that the Karkoschka model leads to a mismatch in shape near $1320 \mathrm{~nm}$, already noted by Tomasko et al. The use of the Irwin et al. (2006) or of our own coefficients solves this problem for the most part. Longwards of $1050 \mathrm{~nm}$, the Karkoschka model is based on the Strong et al. (1993) laboratory measurements, which were restricted to temperatures higher than $190 \mathrm{~K}$. On the other hand, Irwin et al. (2006) derived their coefficients from a joint analysis of the Strong et al. data with more recent measurements of pure methane down to temperatures of $100 \mathrm{~K}$, obtained by Sihra (1998). The much better agreement of the Irwin et al. (2006) model with the Titan spectrum is therefore not surprising, given also the fact that the Sihra measurements included amounts of methane (up to $273 \mathrm{mbar}$ at $100 \mathrm{~K}$ for a cell length of $1326 \mathrm{~cm}$, i.e. a column of $990 \mathrm{~cm}$-am) appropriately similar to that present in the $25 \mathrm{~m}$ spectrum, and a pressure not too different. As discussed above, our coefficients were derived for methane-nitrogen mixtures, and at an effective temperature of $118 \mathrm{~K}$. We therefore show a comparison of our model with two versions of the Irwin et al. (2006) models, respectively at $118 \mathrm{~K}$ and $94 \mathrm{~K}$. The agreement between the three models is generally satisfactory (except for a small deviation at $1100 \mathrm{~nm}$, where our model gives a better match), although curiously, we find that the Irwin et al. model at $94 \mathrm{~K}$ starts to show some deviation with the data near $1340 \mathrm{~nm}$. Note that using a slightly different version of the $25 \mathrm{~m}$ spectrum and the Irwin et al. coefficients, Schröder and Keller (2007) derive a mixing ratio $4.5 \pm 0.5 \%$ from an overall fit of the four methane complexes, and achieve a very good match of the 1150 and $1350 \mathrm{~nm}$ absorptions.

In summary, we find that, in the conditions of Titan's $25 \mathrm{~m}$ spectrum, the Irwin et al. (2006) coefficients provide predictions comparing generally well with our laboratory measurements, and do represent a significant improvement over the Karkoschka model. This however, does not necessarily mean that these coefficients are valid throughout Titan's atmosphere. In fact, while Negrão et al. (2006) found that the Irwin et al. (2006) coefficients also permit a better fit of Titan ground-based spectra compared to the Karkoschka model, Tomasko et al. (2007) point out serious deficiencies of the Irwin et al. coefficients, which must be scaled by factors of $\sim 0.5$ (in band centers) to several (in regions of weak absorption and windows) to fit the ensemble of DISR data. This does not contradict our finding that the Irwin et al. coefficients are adequate to match the "lamp-only" spectra, given that the Tomasko et al. study pertains to either short paths at low pressures (in band centers) or to very long paths (in the methane windows).

\section{Summary}


In order to fully analyze the DISR/DLIS spectra taken during the last phase of the descent of Huygens, we have recorded laboratory spectra in conditions similar to those of Titan's atmosphere. Extrapolating the spectra to the appropriate path lengths, we determine the $\mathrm{CH}_{4}$ mixing ratio to be to $5.1 \pm 0.8 \%$ in the first $25 \mathrm{~m}$ of the atmosphere, and $4.7 \pm 1.0 \%$ for a spectrum taken $90 \mathrm{sec}$ after landing. These figures agree with the initial estimate by Tomasko et al. (2005) ( $5 \pm 1 \%$ for the pre-landing spectrum, then thought to be at $21 \mathrm{~m}$ altitude), based on the "Karkoschka" model, as well as from a more recent determination by Schröder and Keller (4.5 $\pm 0.5 \%)$ from the same dataset, using the Irwin et al. (2006) absorption coefficients. All these numbers agree with the Huygens/GCMS determination of $4.92 \pm 0.25 \%$ near the surface (Niemann et al. 2005). We also infer the surface reflectivity as seen from 25 $\mathrm{m}$ altitude and just after landing, and find a variation in the $1500 \mathrm{~nm}$ band depth.

Although the approach we have used is in principle the most straightforward, the precision of our determination has been in practice limited by the uncertainty in the actual conditions of our low-temperature laboratory spectrum. Ultimately, an accurate modelling of the methane absorptions in Titan spectra will demand a line-by-line knowledge of the methane parameters in the entire near-infrared and visible range. From the experimental as well as theoretical point of view, this is a formidable task. The spectra we have acquired for the present study will contribute to this effort. We plan to complete them by other laboratory measurements, especially in pure methane at low pressures and several low temperatures, and at a even higher spectral resolution.

Ackowledgments: We acknowledge financial support from the French "Programme National de Planétologie". We also acknowledge Stefan Schröder for "illuminating” discussions, and for a digital version of the Surface Science Lamp spectrum. We would like to thank gratefully Michaël Rey, Vincent Boudon and Martin Quack for helpful discussions and giving us recent results that have not yet been published. 


\section{References}

Fulchignoni, M., F. Ferri, F. Angrilli, A. J. Ball, A. Bar-Nun, M. A. Barucci, C. Bettanini, G. Bianchini, W. Borucki, G. Colombatti, M. Coradini, A. Coustenis, S. Debei, P. Falkner, G. Fanti, E. Flamini, V. Gaborit, R. Grard, M. Hamelin, A. M. Harri, B. Hathi, I. Jernej, M. R. Leese, A. Lehto, P. F. Lion Stoppato, J. J. López-Moreno, T. Mäkinen, J. A. M. McDonnell, C. P. McKay, G. Molina-Cuberos, F. M. Neubauer, V. Pirronello, R. Rodrigo, B. Saggin, K. Schwingenschuh, A. Seiff, F. Simões, H. Svedhem, T. Tokano, M. C. Towner, R. Trautner, P. Withers, and J. C. Zarnecki 2005. In Situ measurements of the physical characteristics of Titan’s environment. Nature 438, 785-791.

Hippler, M., Quack, M. 2002. High-resolution Fourier transform infrared and cw-diode laser cavity ringdown spectroscopy of the $v_{2}+2 v_{3}$ band of methane near $7510 \mathrm{~cm}^{-1}$ in slit jet expansions and at room temperature. J. Chem. Phys. 116, 6045-6055.

Irwin, P.G.J., Sromovsky, L.A., Strong, E.K., Sihra, K., Teanby, N.A., Bowles, N., Calcutt, S.B., Remedios, J.J. 2006. Improved near-infrared methane band models and k-distribution parameters from 2000 to $9500 \mathrm{~cm}^{-1}$ and implications for interpretation of outer planet spectra. Icarus, 181, 309-319.

Karkoschka, E., 1998. Methane, ammonia, and temperature measurements of the jovian planets and Titan from CCD-spectrophotometry. Icarus, 133, 134-146.

Karkoschka, E., Tomasko, M.G., Doose, L.R., See, C., McFarlane, E.A., Schröder, S.E., Rizk, B. 2007. DISR imaging and the geometry of the descent of the Huygens probe within Titan's atmosphere. Planet. Space Sci., in press.

McKellar, A.R.W., 1989. The spectrum of gaseous methane at $77 \mathrm{~K}$ in the 1.1-2.6 $\mu \mathrm{m}$ region: A benchmark for planetary astronomy. Can. J. Phys. 67, 1027-1035.

Negrão, Coustenis, A., Lellouch, E., Maillard, J.-P., Rannou, P., Schmitt, B., McKay, C. P., Boudon, V. 2006. Titan's surface albedo variations over a Titan season from near-infrared CFHT/FTS spectra. Planet. Space Sci. , 54, 1225-1246.

Niemann, H. B., S. K. Atreya, S. J. Bauer, G. R. Carignan, J. E. Demick, R. L. Frost, D. Gautier, J. A. Haberman, D. N. Harpold, D. M. Hunten, G. Israel, J. I. Junine, W. T. Kasprzak, T. C. Owen, M. Paulkovich, F. Raulin, E. Raaen, and S. H. Way 2005. The abundances of constituents of Titan's atmosphere from the GCMS instrument on the Huygens probe. Nature $438,779-784$.

Perrin MY, Soufiani A. 2007. Approximate radiative properties of methane at high temperature. Journal of Quantitative Spectroscopy and Radiative Transfer 103,3-13.

Pine AS, Gabard T. 2003. Multispectrum fits for line mixing in the $v_{3}$ band $Q$ branch of methane. Journal of Molecular Spectroscopy 217,105-114.

Prialnik D, Benkhoff J, Podolak, M. 2005. Modeling the Structure and Activity of Comet Nuclei. Comets II, M. Festou, H. U. Keller, H. A. Weaver, eds. University of Arizona Press, 359-387 
Rothman LS, Jacquemart D, Barbe A, Benner DC, Birk M, Brown LR, Carleer MR, Chackerian Jr C, Chance K, Coudert LH, Dana V, Devi VM, Flaud JM, Gamache RR, Goldman A, Hartmann JM, Jucks KW, Maki AG, Mandin JY, Massie ST, Orphal J, Perrin A, Rinsland CP, Smith MAH Tennyson J, Tolchenov RN, Toth RA, Vander Auwera J, Varanasi P, Wagner G. 2005. The HITRAN 2004 Molecular Spectroscopic Database. Journal of Quantitative Spectroscopy and Radiative Transfer 96,139-204.

Schröder, S.E., Keller, H.U. 2007. The reflectance spectrum of Titan's surface at the Huygens landing side determined by the Descent Imager / Spectral Radiometer. Submitted for publication.

Sihra, K., 1998. Laboratory measurements of near-infrared methane bands for remote sensing of the jovian atmosphere. Ph.D. Thesis, University of Oxford.

Sromovsky, L., Irwin, P.G.J., Fry, P.M. 2006. Near-infrared methane absorption in outer planet atmospheres: improved models of temperature dependence and implications for Uranus cloud structure. Icarus, 182, 577-593.

Strong, K., Taylor, F.W., Calcutt, S.B., Remedios, J.J., Ballard, J. 1993. Spectral parameters of self- and hydrogen- broadened methane from 2000 to $9500 \mathrm{~cm}^{-1}$ for remote sounding of the atmosphere of Jupiter. J. Quant. Spectro. Rad. Transf. 50, 363-429.

Tomasko, M. G., D. Buchhauser, M. Bushroe, L. E. Dafoe, L. R. Doose, A. Eibl, C. Fellows, E. McFarlane, G. M. Prout, M. J. Pringle, B. Rizk, C. See, P. H. Smith, and K. Tsetsenekos 2002. The Descent Imager/Spectral Radiometer (DISR) experiment on the Huygens entry probe of Titan. Space Sci. Rev. 104, 469-551.

Tomasko, M.G., Archinal, B., Becker, T., Bézard, B., Bushroe, M., Combes, M., Cook, D., Coustenis, A., de Bergh C., Dafoe, L.E., Doose, L., Douté, S., Eibl, A., Engel, S., Gliem, F., Grieger, B., Holso, K., Howington-Kraus, E., Karkoschka, E., Keller, H.U., Kirk, R., Kramm, R., Küppers, M., Lanagan, P., Lellouch, E., Lemmon, M., Lunine, J., McFarlane, E., Moores, J., Prout, G.M., Rizk, B., Roziek, M., Rueffer, P, Schröder, S.E., Schmitt, B., See, C., Smith, P., Soderblom, L., Thomas, N., West R. 2005. Rain, winds, and haze during the Huygens probe's descent to Titan's surface. Nature, 438, 765-768.

Tomasko, M.G., Bézard, B., Doose, L., Engel, S., Karkoschka, E. 2007. Measurements of methane absorption by the Descent Imager/Spectral Radiometer (DISR) during its descent through Titan's atmosphere. Planet. Space Sci., submitted. 
Table 1. Characteristics of used spectra

\begin{tabular}{|l|l|l|l|}
\hline$\#$ & Mission Time (s) & Altitude (m) & Solar Azimuth $\left(^{\circ}\right)$ \\
\hline 190 & 8712.5 & 708 & 146 \\
\hline 194 & 8825.2 & 200 & 166 \\
\hline 196 & 8832.0 & 169 & 207 \\
\hline 199 & 8845.0 & 109 & 289 \\
\hline 202 & 8850.2 & 86 & 322 \\
\hline 204 & 8853.6 & 72 & 344 \\
\hline 206 & 8857.0 & 55 & 5 \\
\hline 210 & 8863.9 & 24.8 & 45 \\
\hline
\end{tabular}


Fig. 1. Modelling of the room temperature spectrum in the region of the $v_{2}+2 v_{3}$ band. The experimental spectrum recorded at $297 \mathrm{~K}$ with $0.1053 \mathrm{~atm}$ of methane and $0.894 \mathrm{~atm}$ of $\mathrm{N}_{2}$, is plotted in black. In the upper panel, the calculation using all HITRAN transitions is plotted in red, and the one using only assigned transitions of HITRAN is plotted in green. In the lower panel, the calculation using HITRAN* (see text) plus the non assigned transitions from HITRAN is plotted in red, and the one using only HITRAN* is plotted in green. Differences between measurements and models are shown. The lower quantum number $J$ ', for series of transitions of the $v_{2}+2 v_{3}$ band has been indicated on the lower plot. For more visibility, only the even number of $J$ ', have been reported.

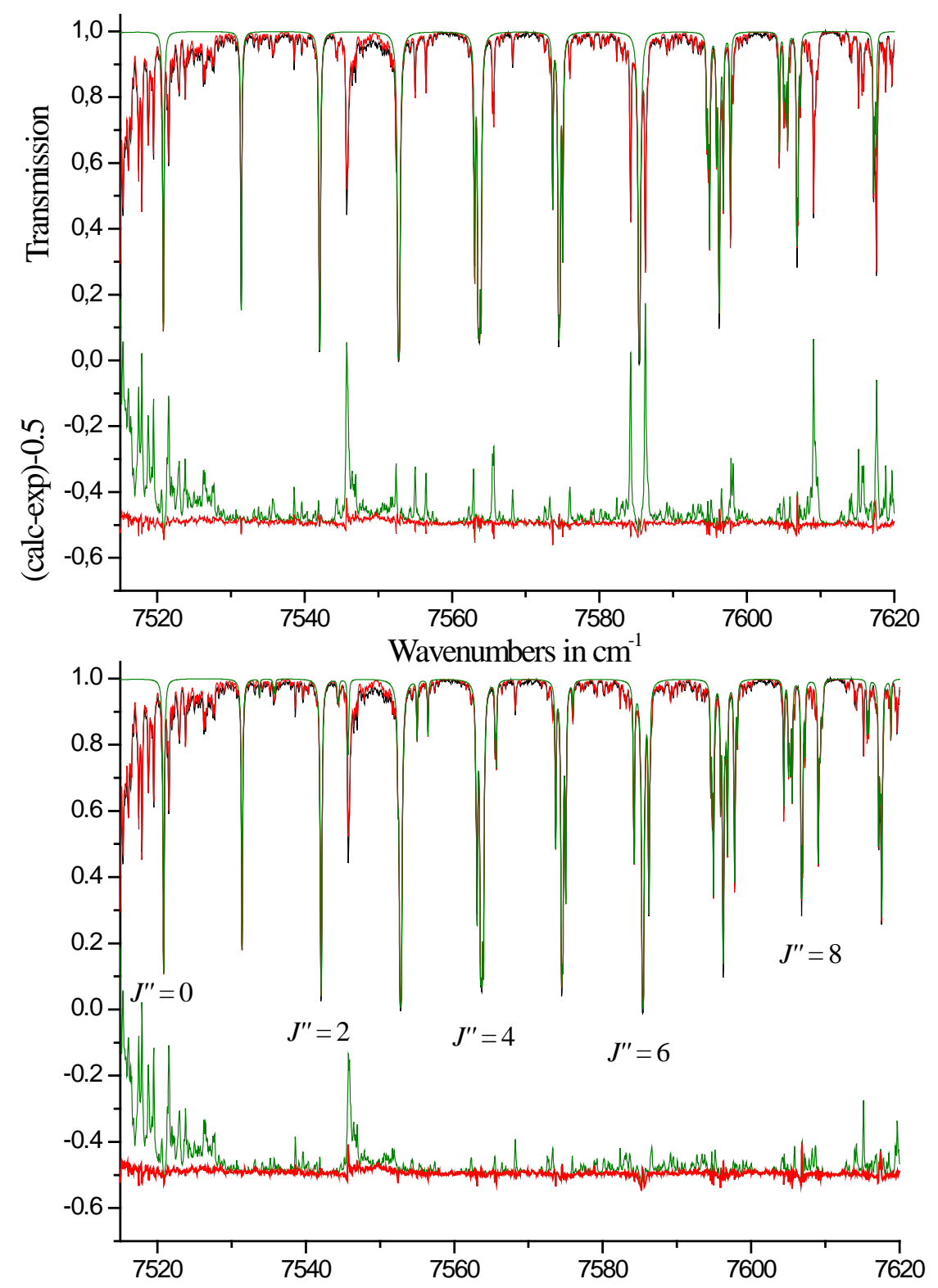


Fig. 2. Modelling of the low-temperature laboratory spectrum (shown in black) at 7515-7620 $\mathrm{cm}^{-1}$. Synthetic spectra of methane broadened by $\mathrm{N}_{2}$ are calculated using HITRAN* (see text) on a grid of temperature and methane column density. The absorption path is equal to 2015 $\mathrm{cm}$, and the pressure of $\mathrm{N}_{2}$ is equal to $1.47 \mathrm{~atm}$. Horizontal lines (from top to bottom) correspond to methane column densities of 158, 178 and $197 \mathrm{~cm}$-am, respectively. Vertical columns (from left to right) correspond to temperatures of 108, 118, and $128 \mathrm{~K}$, respectively. In each panel, the curve at the bottom shows the (obs.-calc.) residuals multiplied by 2. Best fit is achieved for $178 \mathrm{~cm}$-am and $118 \mathrm{~K}$ (central panel).
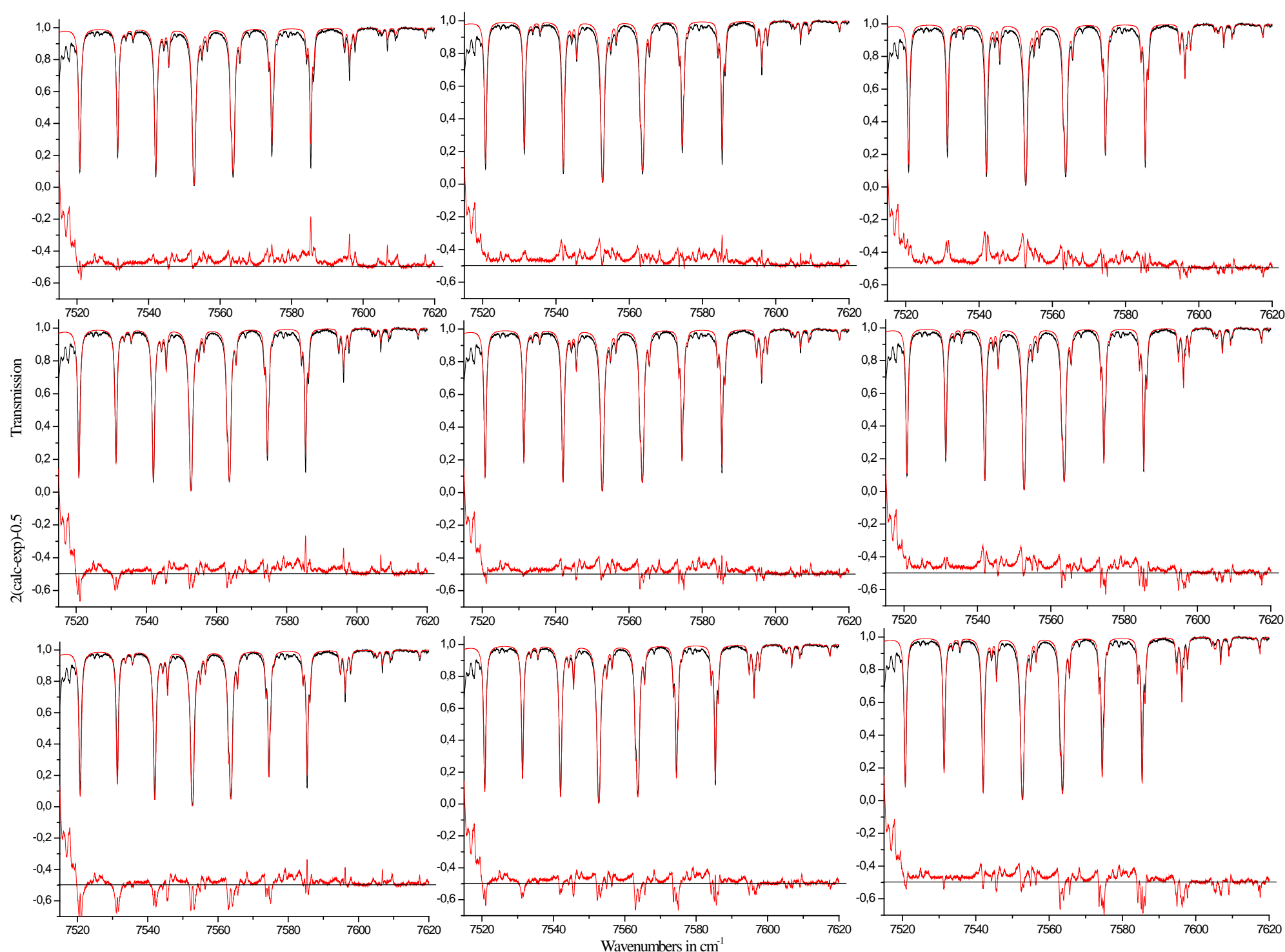
Fig. 3. Low temperature transmission spectrum used to reproduce the DISR/DLIS spectra. The experimental conditions corresponding to this spectrum are $P_{\mathrm{N} 2}=1.47 \mathrm{~atm}, L=2015 \mathrm{~cm}$, $T=(118 \pm 10) \mathrm{K}$, and a methane column density of $178+/-20 \mathrm{~cm}-\mathrm{am}$, i.e. $P_{\mathrm{CH} 4}=(0.038 \pm 0.004)$ atm.

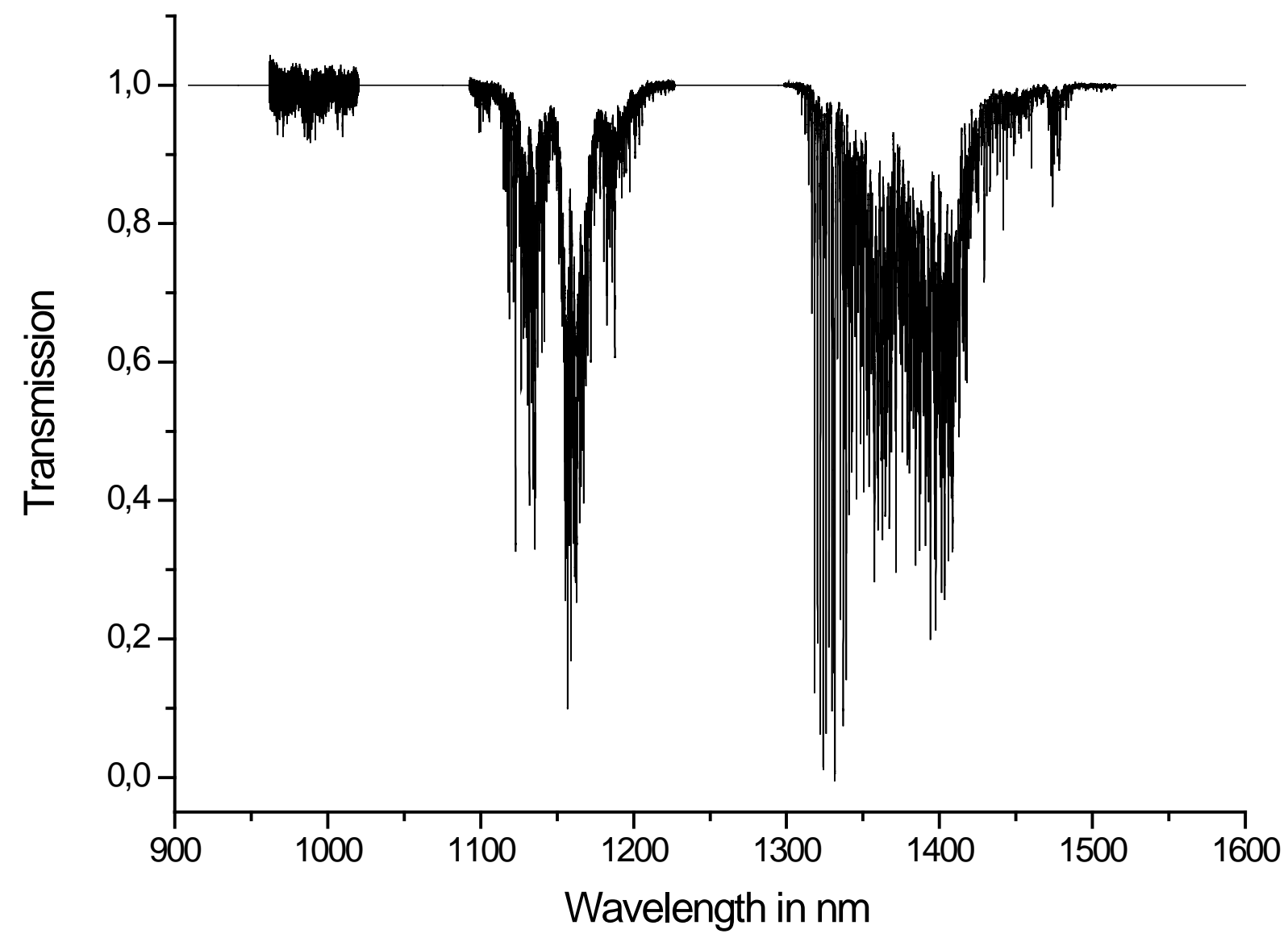


Fig 4. Effect of different choices of "background" spectrum (see text) for constructing the "lamp-only" spectrum at $25 \mathrm{~m}$. "Nominal” refers to \# 210 minus the average of \#199 and \# 202.

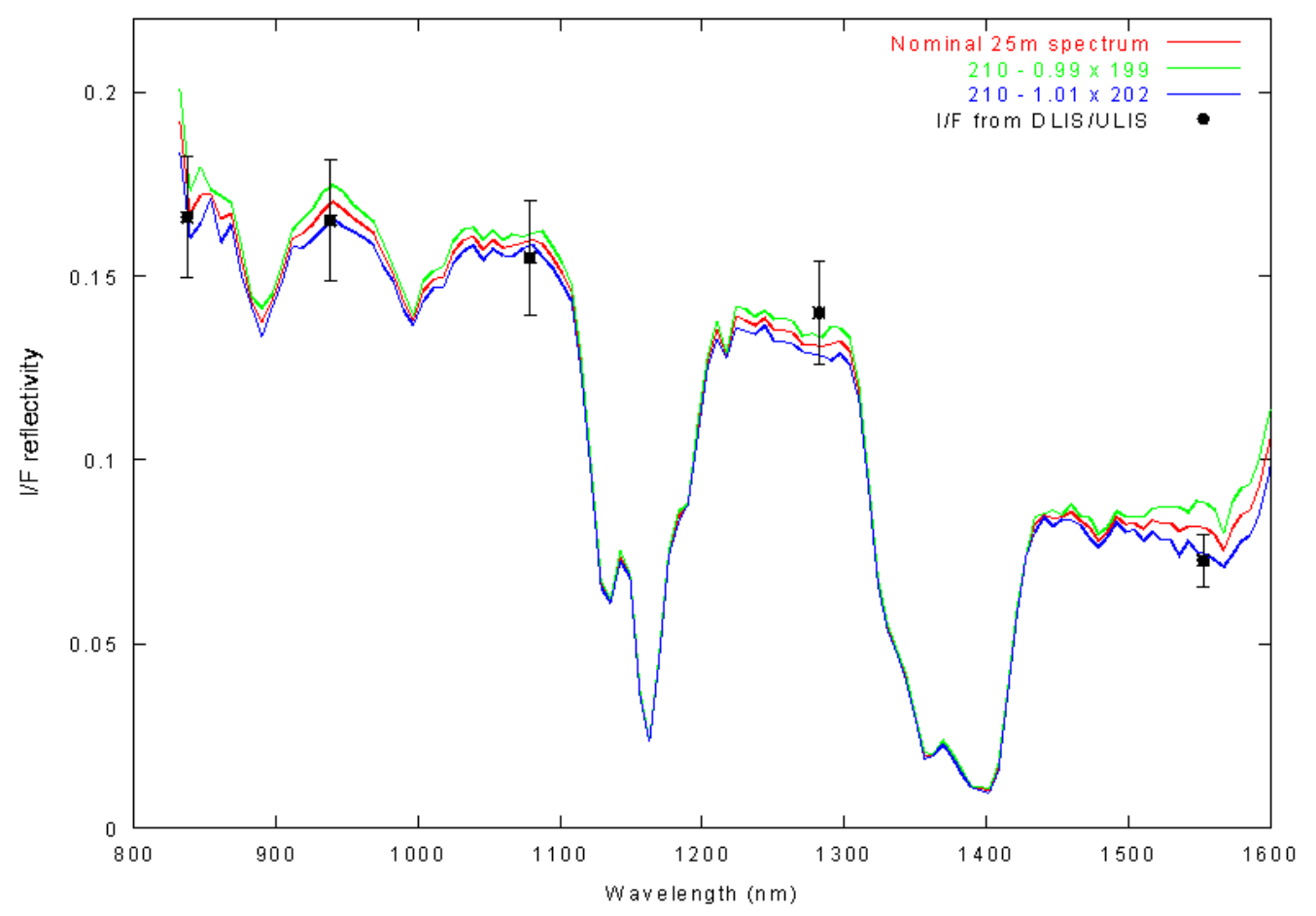


Fig 5. Modelling of the post-landing spectrum in the vicinity of the $1150 \mathrm{~nm}$ methane band. The blue stars show the assumed linear continuum.

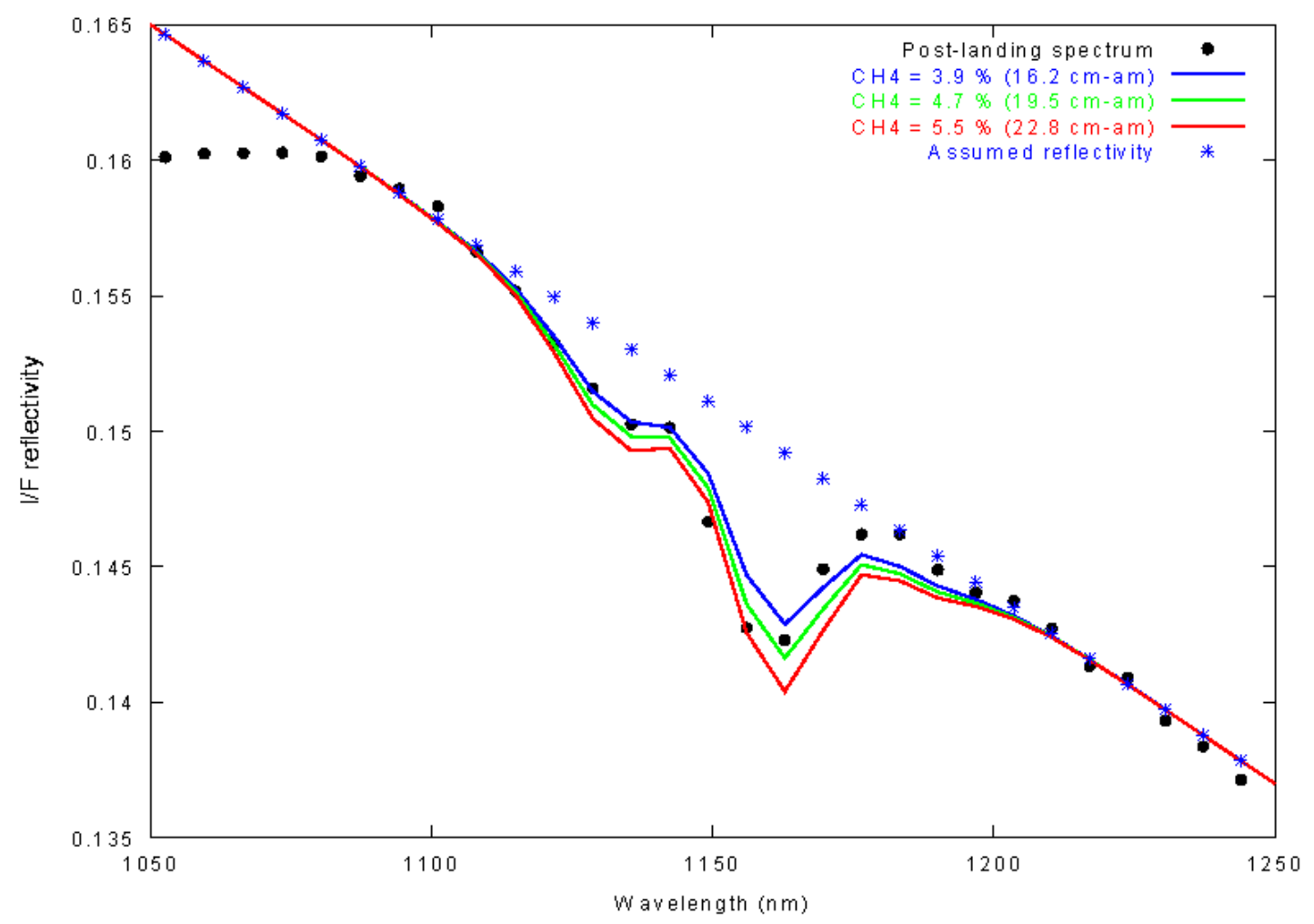


Fig. 6. Comparison of the surface reflectivity inferred from the post-landing spectrum with that used for fitting the spectrum at $25 \mathrm{~m}$.

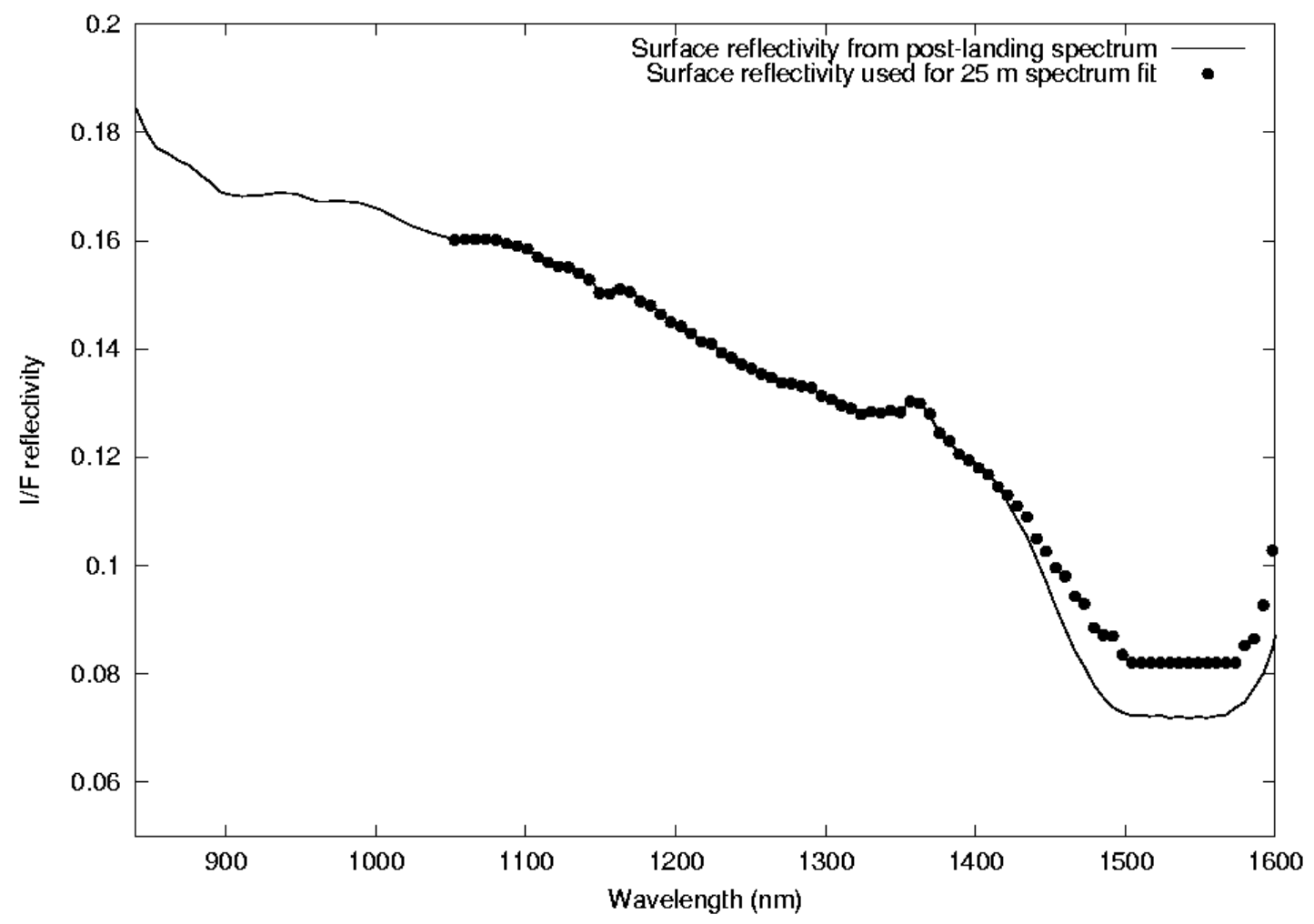


Fig. 7. Modelling of the spectrum at $25 \mathrm{~m}$ for various methane mixing ratios.

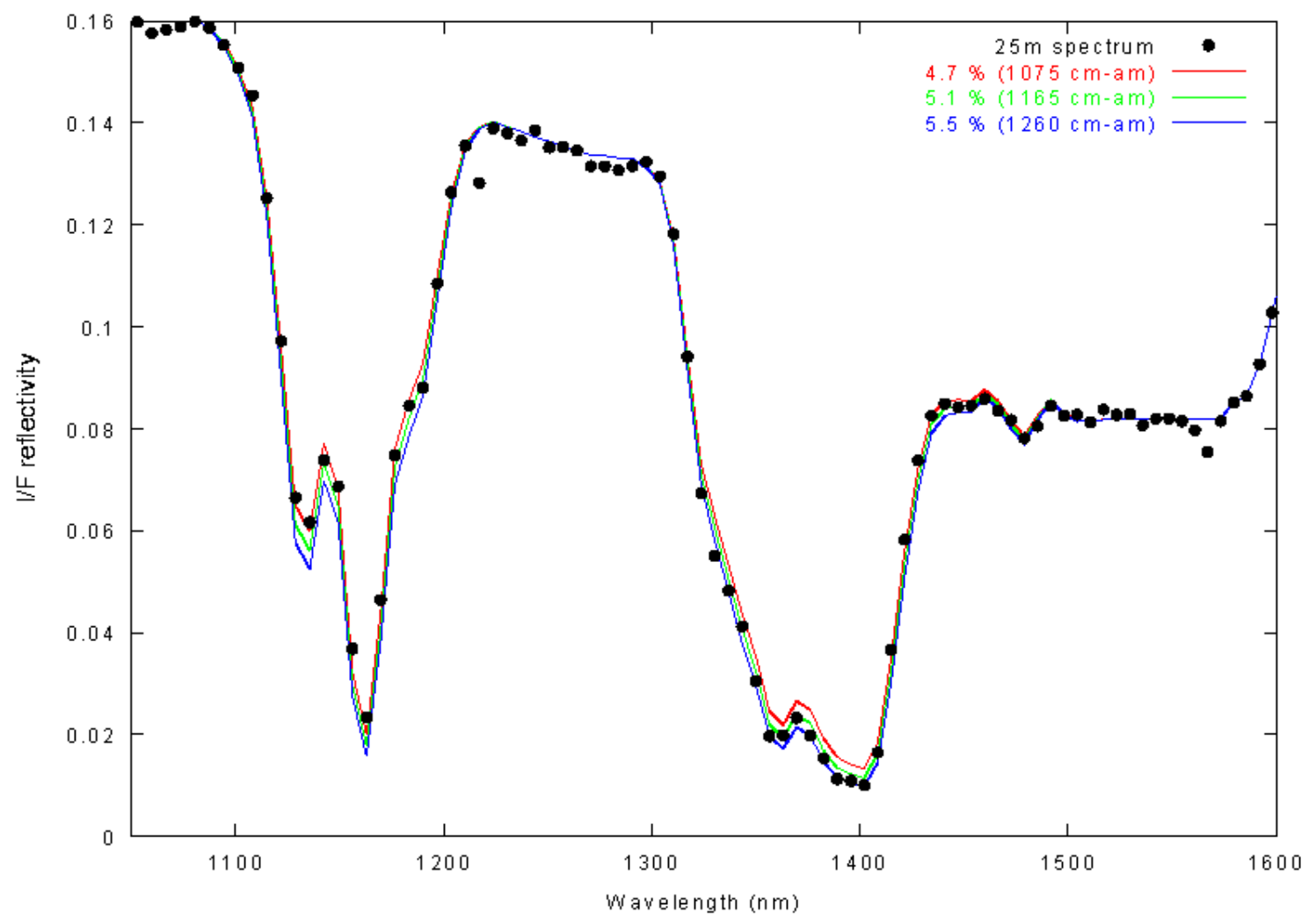


Fig. 8. Synthetic spectra of the pentad (top) and octad (bottom) regions at $118 \mathrm{~K}$ and $94 \mathrm{~K}$. Methane column density and spectral resolution are chosen to roughly match the appearance of the DISR/DLIS spectrum at $25 \mathrm{~m}$.
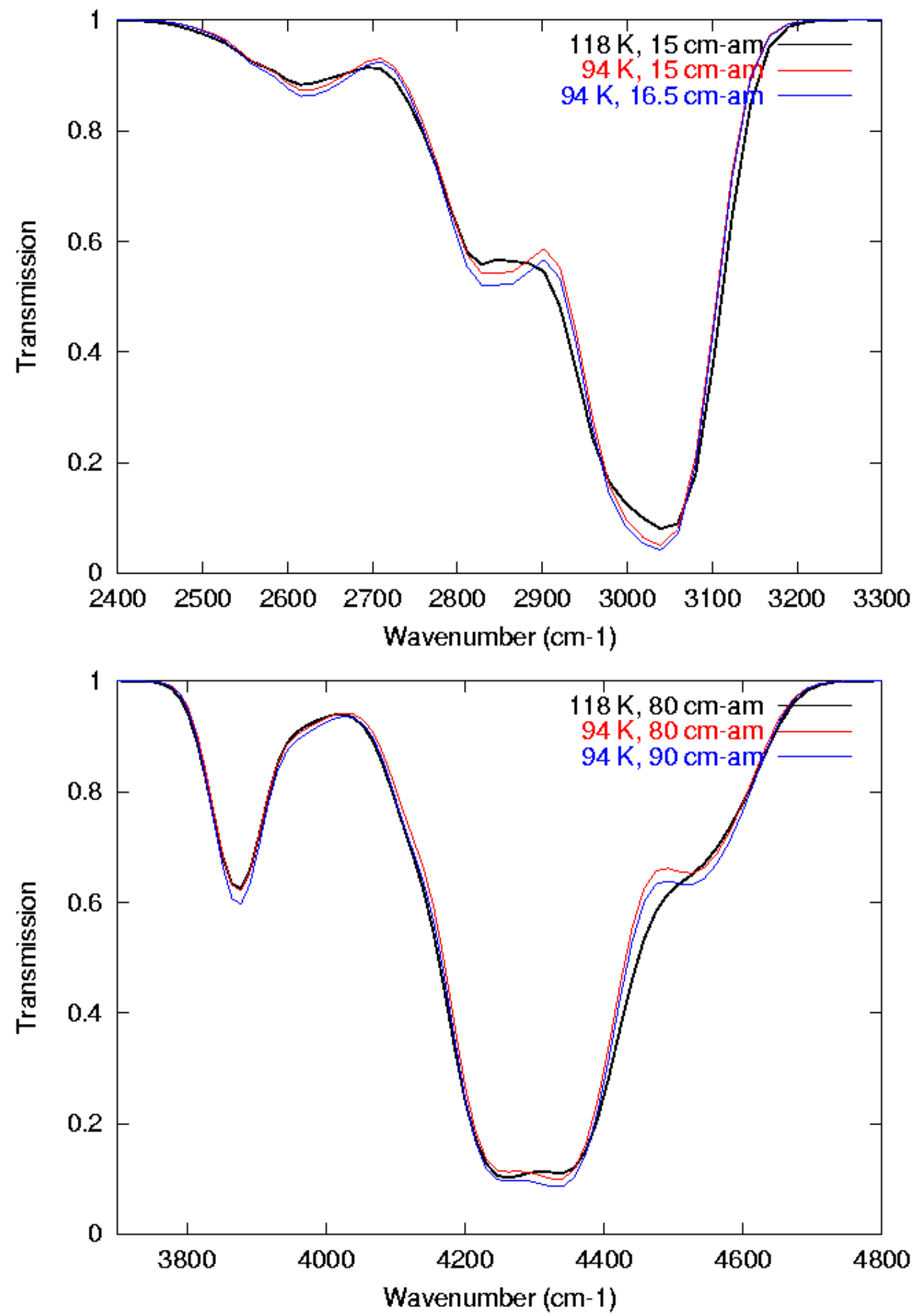
Fig. 9. Fit of the spectrum at $25 \mathrm{~m}$ with various sets of absorption coefficients. The methane column density is $1165 \mathrm{~cm}$-am.

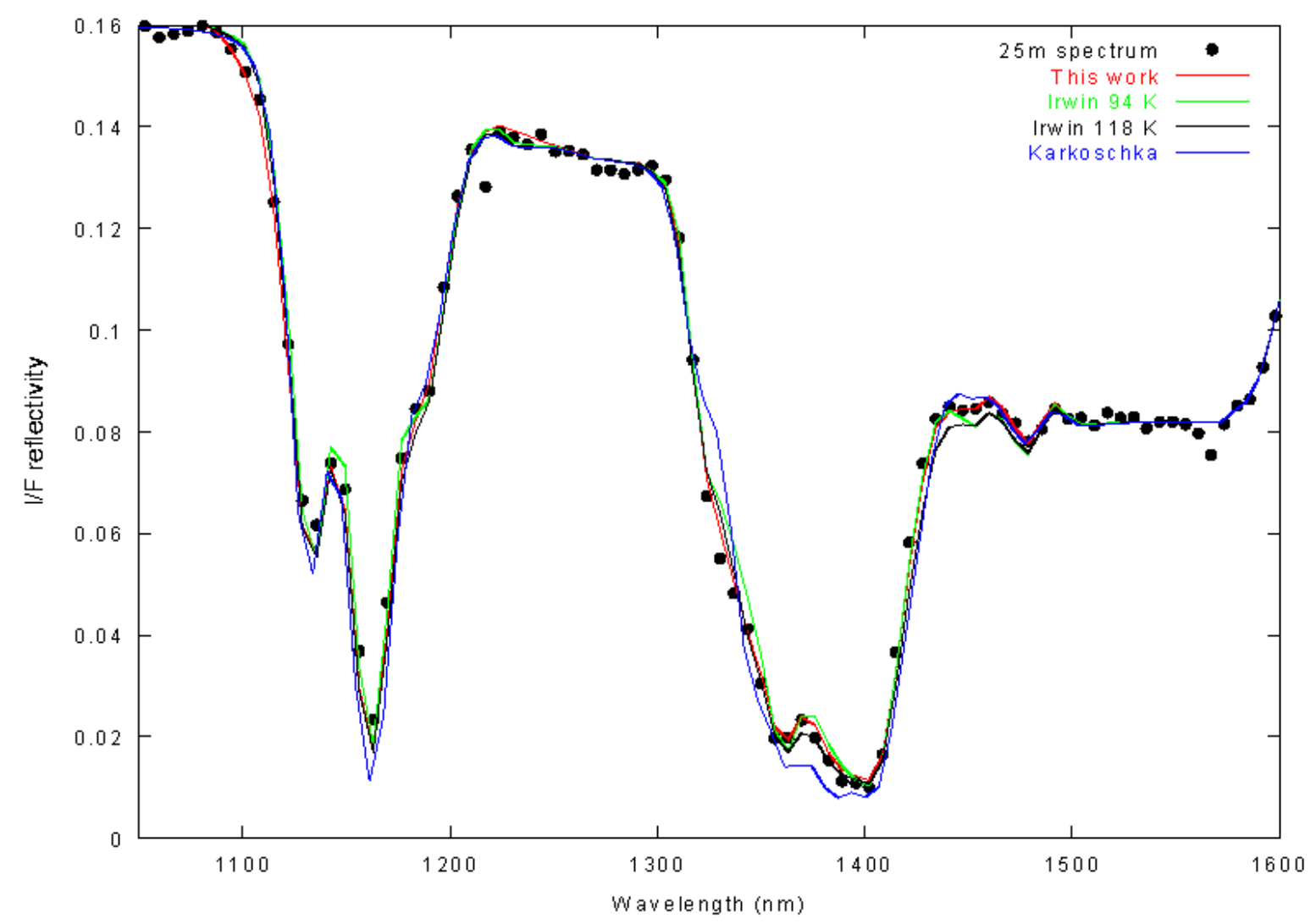

